Annuaire suisse de politique de développement

14 | 1995

Annuaire Suisse - Tiers Monde 1995

\title{
La Suisse et les relations Nord-Sud dans les années 1990
}

Adrian Hadorn

\section{(2) OpenEdition}

Édition électronique

URL : http://journals.openedition.org/aspd/1443

DOI : $10.4000 /$ aspd. 1443

ISSN : 1663-9669

Éditeur

Institut de hautes études internationales et du développement

\section{Édition imprimée}

Date de publication : 1 février 1995

Pagination : 157-160

ISSN : 1660-5934

\section{Référence électronique}

Adrian Hadorn, "La Suisse et les relations Nord-Sud dans les années 1990 », Annuaire suisse de politique de développement [En ligne], 14 | 1995, mis en ligne le 03 mai 2013, consulté le 08 septembre 2020. URL : http://journals.openedition.org/aspd/1443 ; DOI : https://doi.org/10.4000/aspd.1443 


\title{
La Suisse et les relations Nord-Sud dans les années 1990
}

\author{
Adrian Hadorn, Section politique et recherche, DDA
}

\section{Pourquoi une image directrice Nord-Sud?}

Avec son "Rapport sur les relations Nord-Sud de la Suisse dans les années 1990", le Conseil fédéral a rempli un mandat que le Parlement lui avait confié début 1990: présenter "des lignes directrices pour le rôle futur de la Suisse dans la relation Nord-Sud", avec comme objectif "une politique de développement intégrée et cohérente".

Le processus d'élaboration de cette image directrice avait débuté lors des préparatifs de la Conférence de Rio sur l'environnement et le développement de juin 1992 et s'était achevé peu après l'adoption du "Rapport sur la politique extérieure de la Suisse dans les années 1990", en décembre 1993. Ce processus a été influencé par la vision du monde suite à la chute du mur de Berlin en 1989, ainsi que par d'autres événements et les débats internationaux.

Les légitimations géopolitiques du conflit Est-Ouest pour justifer la coopération au développement tombaient du même coup en 1989. La coopération devait brusquement trouver de nouvelles justifications et fut confrontée à une suite imprévisible de malentendus et d'exigences auprès de l'opinion publique et des hommes/femmes politiques:

- ce fut d'abord la déception ressentie par un grande nombre de personne sur la soi-disante inefficacité de la coopération au développement. On parla alors de Aid Fatigue, de phénomènes de lassitude ou d'usure sur le front de l'aide internationale, d'cafricano-pessimisme" ou de la décennie perdue en Amérique latine;

- par ailleurs, la coopération au développement dut faire face à des espoirs totalement irréalisables. On attendait impatiemment d'elle qu'elle puisse résoudre les problèmes du trafic de drogues et des pressions migratoires dans les pays industrialisés. Elle devait aussi éliminer immédiatement dans les pays du Sud les problèmes tels que la surpopulation, les conflits guerriers, la dégradation de l'environnement, le surendettement et d'autres crises économiques, ainsi que la corruption et les autres formes de gestion gouvernementale inefficace. D'autres priorités (aide aux pays de l'Est) vinrent supplanter ces préoccupations au même moment. 
Des expériences multiples et concrètes de coopération en Afrique, Asie et en Amérique latine montraient en outre que certains projets de développement n'avaient eu malgré le professionnalisme avec lequel ils avaient été planifiés et exécutés que peu d'effets, voire aucun effet positif. Ces échecs survenaient dès lors que les conditions-cadres économiques, sociales ou politiques étaient inappropriées.

Enfin, les énormes déficits budgétaires qu'accusèrent de nombreux pays industrialisés renforcèrent les positions de ceux qui réclamaient une révision de la coopération au développement, son intégration plus effective dans le contexte général de la politique extérieure et de la coopération internationale, ainsi qu'une utilisation plus efficace des ressources financières limitées pour la coopération Nord-Sud.

En Suisse, la politique étrangère est devenue, comme jamais auparavant, une pomme de discorde. Les tensions et les incertitudes sur la voie, le but et l'identité de la Suisse dans un environnement qui évolue très vite marquent profondément la manière helvétique d'appréhender le monde actuel.

\section{Pourquoi la "cohérence» est-elle un thème permanent dans l'image di- rectrice Nord-Sud?}

Peter Maurer présente dans son article ce que veut dire cohérence dans le "Rapport sur la politique étrangère de la Suisse dans les années 90». Cette précision pragmatique est nécessaire, car la conception d'une "politique étrangère et de développement cohérente" risque facilement de devenir une formule creuse. A cette conception positive et orientée sur l'action, qui prône une optimisation de l'activité politique globale (policy mix), j'aimerais mentionner une dimension qui préoccupe de plus en plus la population en Suisse, mais aussi ailleurs; la dimension de l'interdépendance. Cette interdépendance fait de notre politique étrangère un élément de la politique mondiale.

$\mathrm{Si}$, par exemple, de nouveaux Tchernobyl se produisaient, la coopération Est-Ouest pourrait représenter la sauvegarde des intérêts vitaux ou une condition même de la survie de l'Europe de l'Ouest. Si la forte croissance démographique persiste dans le Sud, si elle provoque des migrations et menace la sécurité dans diverses régions du monde, nos buts de politique étrangère ne seront réalisables que si nous réussissons à instaurer une meilleure solidarité internationale. Si notre mode de consommation est imité intégralement dans un certain nombre de pays au seuil de la pauvreté (en Indonésie, Chine, Inde ou au Brésil par exemple), les effets négatifs de la croissance sur l'environnement seront de plus en plus sensibles et auront des conséquences sur notre politique intérieure et extérieure. En d'autres termes: la question de la cohérence est inscrite dans les problèmes réels et ces interdépendances compliquent l'action politique. II ne suffit plus de dire "gouverner, c'est prévoir", car au prévoir s'ajoute la nécessité d'“agir par avance", ce qui implique que l'action politique doit intégrer les effets imprévisibles d'interactions sur l'environnement, en vue d'un développement durable. 
Nos modèles courants d'analyse politique ne sont pas suffisamment préparés à pareille situation, ni en Suisse, ni dans le reste de l'Europe, ni dans d'autres régions du monde, ni dans les instances multilatérales (ONU ou Banque Mondiale).

Au cours des prochaines années et décennies, les responsables politiques devront revoir de manière fondamentale leurs politiques, sous la pression de la société civile qui subit de plus en plus les conséquences des interactions et de l'interdépendance. Faute de quoi, dans seulement un siècle, "gouverner" voudra seulement dire "avoir prévu que cela devait se passer ainsi...".

L'image directrice Nord-Sud n'est de loin pas aussi futuriste. II suffit d'en parcourir le texte pour se rendre clairement compte qu'elle ne constitue pas une potion magique contre toutes les contradictions et les incohérences des relations Nord-Sud. Elle s'attache plutôt à ébaucher des champs d'action qui se situent aux intersections entre les différents objectifs suivants:

- sécurité - justice sociale

- environnement - justice sociale

- justice sociale - démocratie

L'image directrice offre le cadre d'un programme d'action politique qui inclut tous les acteurs du domaine public et privé (non étatique). Les éventuelles contradictions entre les intérêts nationaux à court terme et les objectifs de la politique de développement suisse doivent être mises en évidence et intégrées avec le maximum de transparence dans le processus de décision politique.

\section{Comment appliquer l'image directrice?}

Définir des champs d'action et postuler la cohérence sont une chose. Agir en conséquence et opérer des changements en sont une autre. L'heure de vérité pour l'image directrice n'a pas été le débat au Parlement. Elle ne sonnera que lors des décisions futures qui seront prises à l'intérieur des différents conflits entre objectifs mentionnés ci-dessus.

Le colloque sur les questions de cohérence de nos relations bilatérales avec I'Indonésie, organisé en commun par l'IUED avec la Communauté de travail des organisations d'entraide et l'administration fédérale (DDA et OFAEE) a ouvert des options pour une action politique différente sur deux plans:

- De prime abord, I'Indonésie n'est aucunement d'intérêt vital pour la Suisse. C'est un grand pays doté du potentiel d'un nouveau "dragon" dans le contexte extrêmement dynamique du sud-est asiatique. Mais c'est en même temps un pays plein d'inconnues pour l'avenir: risque d'instabilité politique, justice sociale, commercialisation durable de ressources naturelles limitées. Comment notre policy mix doit-il s'articuler? Les exposés de la DDA et de l'OFAEE ci-après ébauchent de telles possibilités d'optimisation. Des possibilités s'offrent à nous de conduire une politique de développement où la Suisse rassemble, au niveau de ses relations avec 
d'importantes régions, toutes les interactions significatives dans une même image globale. Sur cette base, la science, l'économie et d'autres acteurs privés, sans oublier le secteur public (y compris la responsabilité qu'il exerce pour notre politique dans les organisations internationales telles que la Banque mondiale, le GATT ou le programme démographique de l'ONU) définissent leur propre échelle de priorités et leurs programmes d'action.

- Il ressort des discussions qui se sont déroulées au cours de ce colloque sur l'Indonésie que tous les protagonistes - qu'ils appartiennent aux milieux scientifiques, économiques, aux associations d'entraide ou à l'administration - pensent tirer un profit pour eux-mêmes, dès lors qu'un accord est trouvé sur les objectifs généraux et que l'on a atteint une certaine clarification des rôles dans le dialogue. A cet égard, il ne s'agit en aucun cas que "tous aillent dans la même direction", comme l'a estimé un représentant de l'économie. Mais il est incontestable que précisément la clarification des rôles dans l'économie privé constitue une tâche cruciale sur le plan de la politique de développement. Cette tâche, il nous reste à l'accomplir et, pour ce faire, à gagner à notre cause des alliés provenant des milieux les plus divers. 